\title{
Speech Aid Prosthesis: A Non-surgical Approach for the Management of Velopharyngeal Insufficiency
}

\author{
${ }^{1}$ Purnendu Bhushan, ${ }^{2}$ Tapan K Patro, ${ }^{3}$ Angurbala Dhal, ${ }^{4}$ Lokanath Garhnayak, ${ }^{5}$ Debasish Mishra, ${ }^{6}$ Arunkharavela Mohanty
}

\section{ABSTRACT}

Aim: Managing a case of velopharyngeal insufficiency (VPI) using speech aid prosthesis (SAP) fabricated using computerassisted designing (CAD)-computer-assisted manufacturing (CAM) laser sintering technology.

Background: Velopharyngeal insufficiency (VPI) is a failure of the body's ability to temporarily close the communication between the nasal cavity and the mouth because of an anatomic dysfunction of the soft palate or the lateral or posterior wall of the pharynx.

Velopharyngeal insufficiencies (VPIs) can be congenital or acquired which may require surgical intervention. However, when the surgical treatment is not feasible or opted (due to various reasons), by a patient, prosthetic management of velopharyngeal insufficiency can be carried out non-surgically using a speech-aid prosthesis.

Case description: A single case, where a 37-year-old male patient reported with the complaint of inadequate clarity of speech due to the defect in the palate since birth. The patient exhibited symptoms of velopharyngeal insufficiency. The patient was not motivated to undergo surgery and also had associated medical contraindications due to uncontrolled diabetes. To manage this case a speech aid prosthesis (SAP) with speech bulb on a Co-Cr metal framework was fabricated with the use of CAD-CAM laser sintering.

Conclusion: A logical approach to the history taking and treatment planning resulted in a successful outcome for the patient, using speech aid prosthesis. In the current case, there was an appreciable improvement in the ease of speech and its clarity for the patient.

Clinical significance: The CAD-CAM laser sintering fabricated speech aid prosthesis helps in better management of velopharyngeal insufficiency with enhancing phonetics and muscle response.

Keywords: Computer-assisted designing-computer-assisted manufacturing, Laser sintering, Speech aid prosthesis, Velopharyngeal insufficiency.

\footnotetext{
${ }^{1,6}$ Department of Prosthodontics, Kalinga Institute of Dental Sciences, KIIT University, Bhubaneswar, Odisha, India

${ }^{2,3}$ Department of Prosthodontics, Sriram Chandra Bhanj Government Dental College and Hospital, Cuttack, Odisha, India ${ }^{4}$ Department of Prosthodontics, Sriram Chandra Bhanj Government Dental College and Hospital, Bhubaneswar, Odisha, India

${ }^{5}$ Department of Periodontics and Oral Implantology, Kalinga Institute of Dental Sciences, KIIT University Bhubaneswar, Odisha, India

Corresponding Author: Debasish Mishra, Department of Periodontics and Oral Implantology, Kalinga Institute of Dental Sciences, KIIT University, Bhubaneswar, Odisha, India, email: dr.deb@zoho.com
}

How to cite this article: Bhushan P, Patro TK, Dhal A, Garhnayak L, Mishra D, Mohanty A. Speech Aid Prosthesis: A Non-surgical Approach for the Management of Velopharyngeal Insufficiency. World J Dent 2019;10(1):67-71.

\section{Source of support: Nil}

Conflict of interest: None

\section{BACKGROUND}

The speech production is a sophisticated mechanism, which is the result of coordinated physiological actions that include respiration, phonation, resonation, articulation and neurological integration. ${ }^{1,2}$

The ability for speech comes from the power generated through expiratory air. This pressure goes through the larynx and vibrates the vocal cords, which leads to phonation.

Various resonators, e.g., pharynx, oral cavity, nasal cavity, paranasal sinuses, modulates the sound produced by the vibration of vocal cords.

After a series of sequential events the voice is transformed into meaningful words is by the correct sequential action of tongue, teeth, lips, palate, and mandible.

Palatopharyngeal or velopharyngeal (VP) closure mechanism is responsible for speech creation along with other physiological oral activities such as whistling, blowing, swallowing and sucking.

Any malfunctioning in the velopharyngeal mechanism (VP) results in conditions such as hypernasality, inappropriate nasal escape, and reduced or inadequate air pressure during the production of oral speech sounds. ${ }^{3,4}$

The velopharynx is a muscular valve placed at the junction between the oral and nasal cavities. It consists of two main components-lateral and posterior pharyngeal walls and the soft palate which controls the passage of air. ${ }^{5}$

Due to the lack of velopharyngeal movement (also known as velopharyngeal incompetence or dysfunction), the velopharynx valve is not able to complete its timely closing to control air passage. ${ }^{6}$

The deficiencies of the soft palate are grouped into either congenital or acquired defects. In the cases of the congenital cleft palate, the development and growth of the hard and soft palate are interrupted at the embryological stage.

There are also cases of VP malfunctioning that occur after the surgical removal of neoplastic growths. The surgical process results in disturbing the soft palate continuity, which leads to an acquired defect. 
Another set of reasons for the reduced dexterity of the soft palate to complete the physiological actions of the oral cavity can be underlying neurologic muscular weaknesses.

In the clinical cases of velopharyngeal insufficiencies, obturator prosthesis can be used to provide a timely closing of the opening between the hard/soft palate and the pharynx.

Speech aid prosthesis (SAP) is a pharyngeal obturator which extends beyond the residual soft palate to prosthetically create the required separation between oropharynx and nasopharynx. It provides a stable structure against which the muscle of the pharynx can move to form the palatopharyngeal closure.

The velopharyngeal incompetence which is due to a neuromuscular disorder or muscle weakness is managed with palatal lift prostheses (PLP). It limits the hypernasality by bringing the incompetent soft palate and the posterior wall of the pharynx closer to each other. ${ }^{6}$

Speech aid prosthesis (SAP) consists of the functional portion called the speech bulb or the nasopharyngeal section that is shaped suitably to support the action of VP during speech and swallowing.

The success of the SAP depends on multiple factors such as location, type, comfort and proper fitting of a prosthesis. The efficiency of the SAP also hinges on the age when the defects originate and also on adaptability to the prosthesis.

Subjects with congenital VP incompetency go through a long stretch of time without learning speech phonation. Therefore, in such scenarios, where the patient has not adapted with the speech phonation since the beginning, it is always a challenge to learn and get used to proper phonation even with the use of SAP. It needs a prolonged period of adjustment and learning before the subject gets habituated to generate phonation with the help of SAP.

\section{CASE DESCRIPTION}

A 37-year-old male patient with congenital palatal defect reported with a complaint of poor clarity of his speech.
Occlusion was class I standard type with healthy gingiva and periodontium.

On clinical examination, he was found to have congenital midline cleft palate of size $6 \times 8 \mathrm{~mm}$ which was restricted only to soft palate involving the posterior border (Fig. 1). He exhibited the signs of velopharyngeal dysfunction and was interested to improve the speech clarity.

The patient was thoroughly informed about the various treatment modalities available including the surgical approach. However, he was not interested to undergo a surgical procedure for the management of speech insufficiency. He, however, agreed to initially undergo speech correction via the use of a prosthesis. Therefore, the treatment plan involved an SAP with a speech bulb on a cast metal framework. The process was explained to the patient in entirety, and his consent was taken.

\section{Clinical Procedure}

The palatal defect was blocked with gauze piece-coated with petrolatum jelly. The primary impression of the maxillary arch was made using irreversible hydrocolloid impression material (Fig. 1).

An impression was poured using dental stone type III, and the original cast was obtained. Custom tray extending till the defect was fabricated on the primary cast with autopolymerizing acrylic resin blocking the area of teeth with wax. The designing of the prosthesis was done on the primary cast after completing surveying (Fig. 2A).

After occlusal rest preparation, a secondary impression of the maxillary arch was made with a custom tray using medium body polysiloxane material. This impression was poured with a type IV gypsum product. CAD of the framework was done after scanning of the master cast (Fig. 2B). This design consists of metal extension posteriorly from posterior palatal strap for speech bulb.

Laser sintered $\mathrm{Co}-\mathrm{Cr}$ metal framework fitting was checked on master cast. After metal finishing and
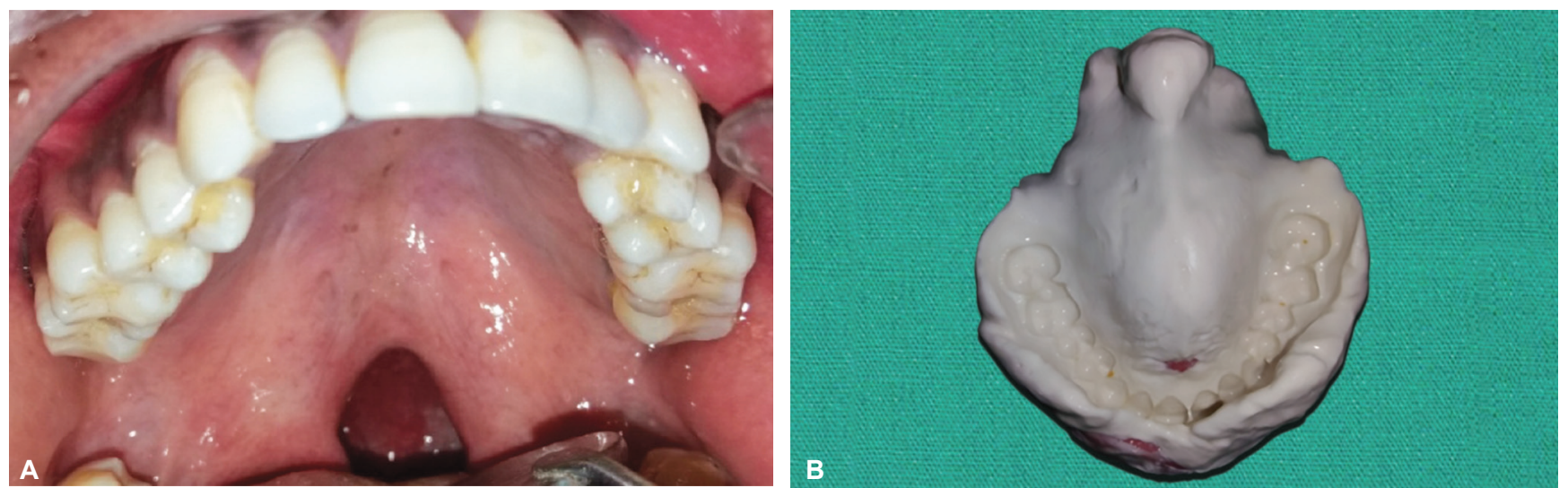

Figs 1A and B: (A) Intraoral view of cleft palate; (B) Secondary impression 
polishing, metal framework try-in was carried out in the patient's mouth for confirming proper fit and retention (Fig. 3).

A functional impression of the defect for proper velopharyngeal closure was taken by keeping impression material over metal extension mesh (Fig. 4A).

First, the gross defect area was recorded with a high fusing impression compound. As these are usually overextended, so it required scrapping and functional border molding of the defect with low fusing green stick compound while patient swallows speaks, makes a circular head movement and breathes appropriately. Final functional contouring of defect was done by placing a layer of soft resilient tissue conditioning material. This functional impression was attached to master cast with altered cast technique and pouring of defect portion was done with the type III gypsum product. After the final setting of material, the cast was placed in hot water for removal of the impression (Fig. 4B).

The defect area on master cast coated with a separating medium was poured with autopolymerizing resin till the superior border of defect extension. Now metal framework was placed over master cast, care was taken that auto-

polymerizing resin should come out through metal mesh. SAP on the master cast was placed in pressure pot with water for 15 minutes at 30 psi. (Fig. 5)

Finishing and polishing of prosthesis were done after recovering it from the mold. On insertion, evaluation of the obturator and the speech bulb was done for proper border extensions using pressure indicating paste (Figs $6 \mathrm{~A}$ and $\mathrm{B})$.

\section{DISCUSSION}

The role of the soft palate is to maintain the functional separation between the oral and nasal cavities. To fulfill this role, the soft palate undergoes synchronized movements as per the physiological demands of speech, respiration, and deglutination. This coordinated soft palate movement leads to the required degree of closure between the pharyngeal walls and soft palate.

To produce phonation for vowels and most of the consonants, the velopharyngeal wall needs to be closed completely. In contrast, the VP valve needs to be completely open for the production of nasal sounds $(\mathrm{m}, \mathrm{n}, \mathrm{ng}){ }^{8}$

The defectsin the softpalate (congenitalor acquired) lead to the continuity between oropharynx and nasopharynx.
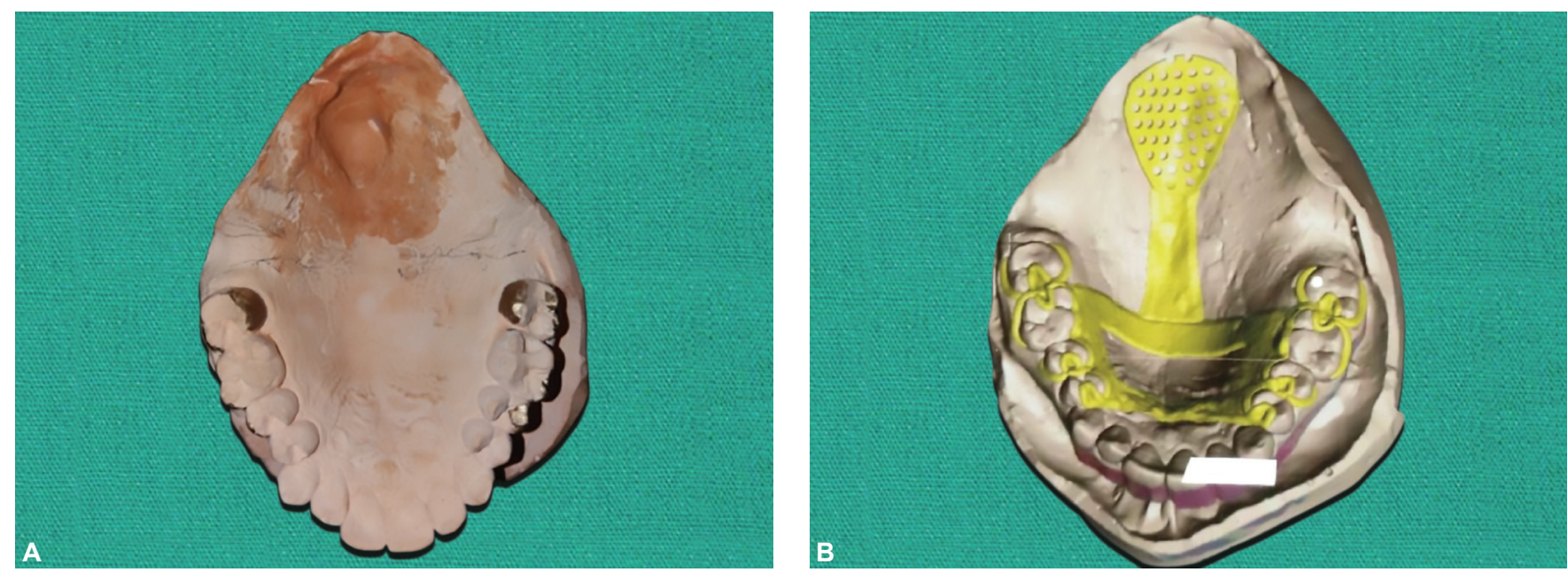

Figs 2A and B: (A) Master cast; (B) Computer-assisted design for laser sintering of framewok
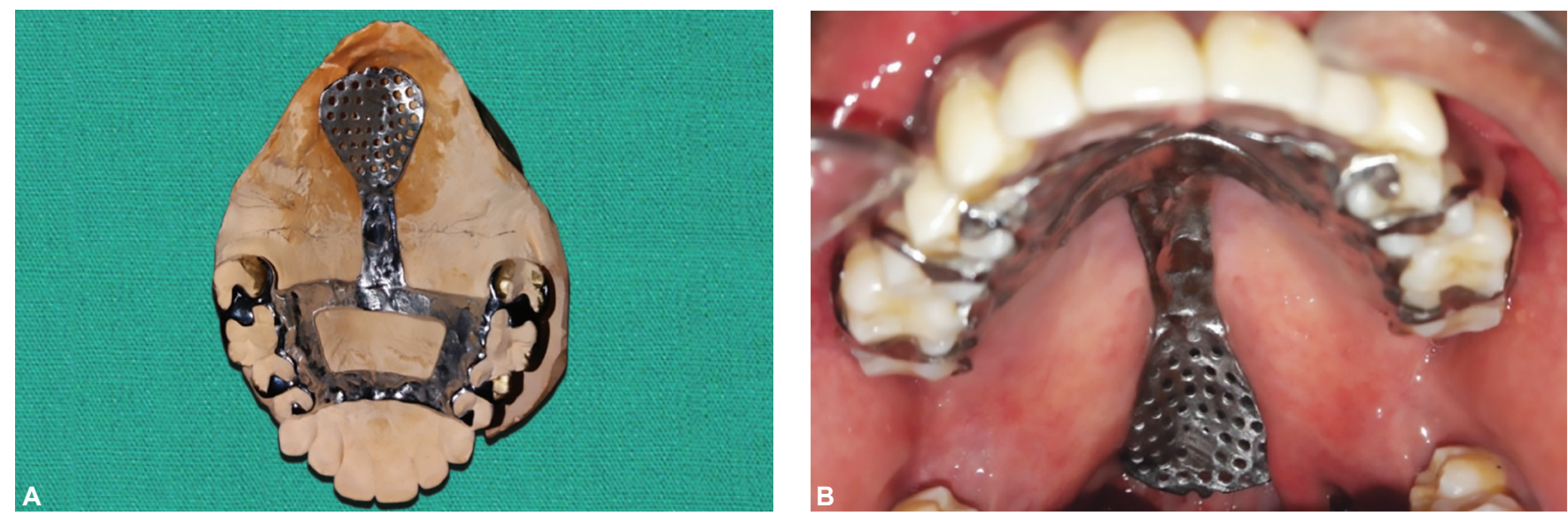

Figs 3A and B: (A) Metal framework after finishing and polishing; (B) Metal framework try-in 

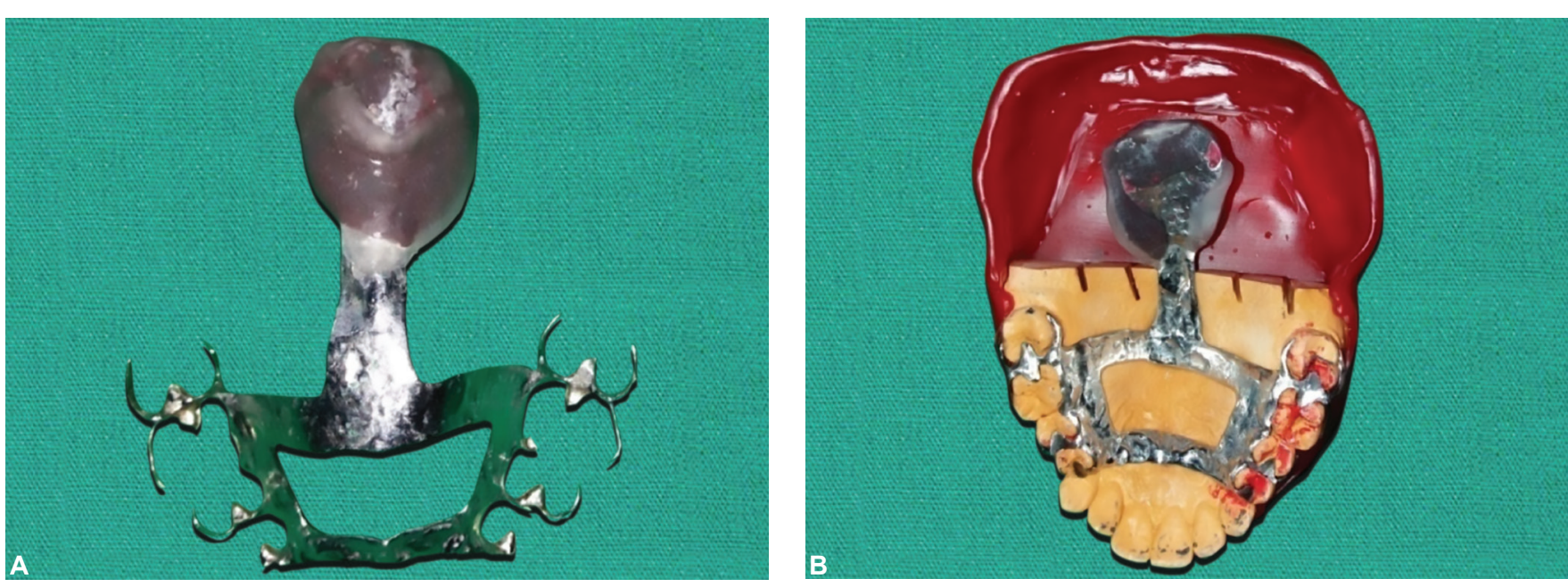

Figs 4A and B: (A) Functional impression; (B) Altered cast technique
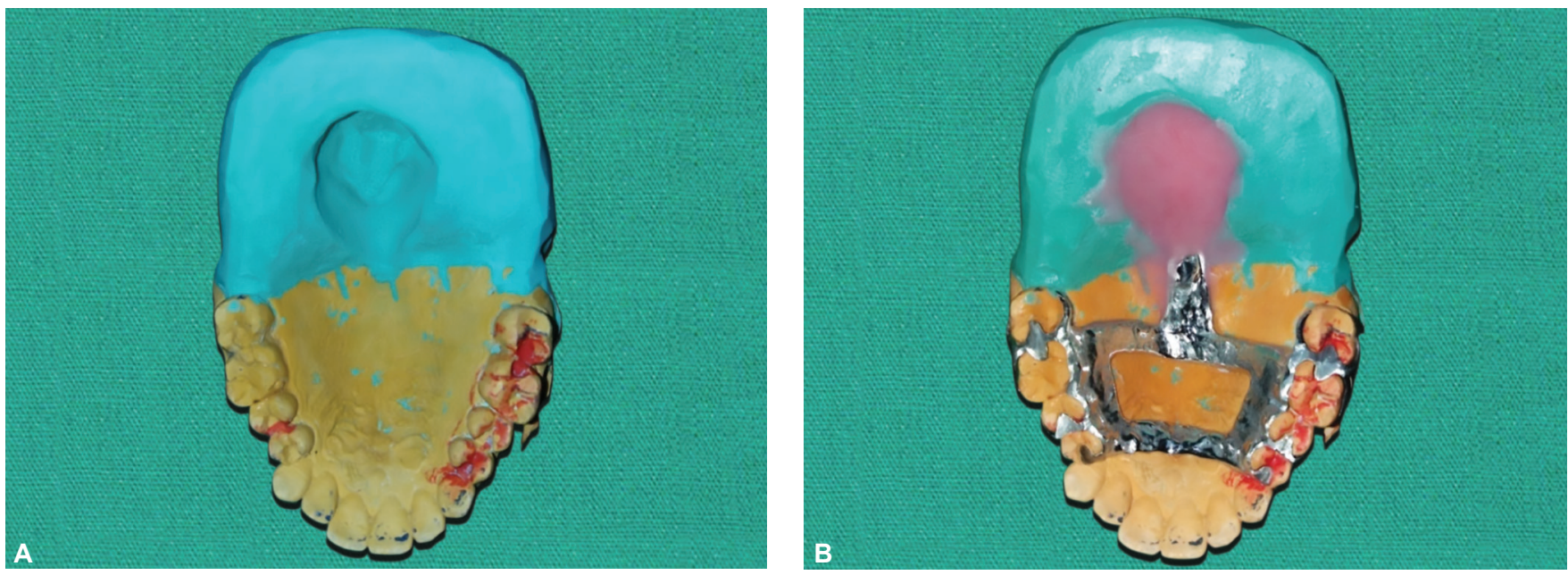

Figs 5A and B: Altered master cast; (B) Speech bulb fabrication on altered master cast

The primary goal of the prosthetic intervention is to prevent the regurgitation of fluid and food along with improving the speech. ${ }^{9}$ Properly obturated defects reduce the nasalance or hypernasality of voice and hence, speech becomes more clear.

From a management point of view, a complete soft palate defect is relatively easier to manage with a better outcome than a partially resected or dysfunctional soft palate. During the treatment, the exact level of the obturator prosthesis in the nasopharynx is affected by the position and efficiency of the velopharyngeal mechanism.

In subjects with incompetent soft palates, the point of insufficient closure should extend to around 5-7 mm of vertical height, to establish an effective closure at the level of the palatal plane and anterior tubercle of the atlas. Hence, it is essential that in the tracing procedure, there is no overextension or underextension, to achieve the accurate tissue approximation. ${ }^{10,11}$

In classic prosthodontic literature, House provides three classifications of soft palates.

In his literature, he has stated that, in the context of a complete denture therapy, the most viable soft palate orientation is when it extends horizontally from the hard palate. The least desired scenario is when the soft palate is nearly at an angulation of 90 degrees to the plane of the hard palate. ${ }^{12}$

In the context of the present case report, it is much easier and efficient to obturate an incompetent soft palate with more horizontal orientation. Normal casting procedure for long-span bridges or cast partial dentures has its disadvantages due to metal shrinkage and complex procedures, which could be overcome with new CAD-CAM technology. A metal framework designed with software and laser sintered was precise fit and mechanically stable. ${ }^{13}$ This DMLS process is carried out by forming a thin layer of metal powder (ranging from 20 to $100 \mu \mathrm{m}$ ) and sintered by a laser(high powered ytterbium fiber laser) to form a 2D cross-section. This is then built up layer-by-layer by distributing an even layer of metallic powder to produce the 3D object.

One of the significant portions of the treatment planning is to reduce the apprehension of the patient and reassurance that there will be an improvement in the speech. It is also imperative to motivate the patient regarding his 

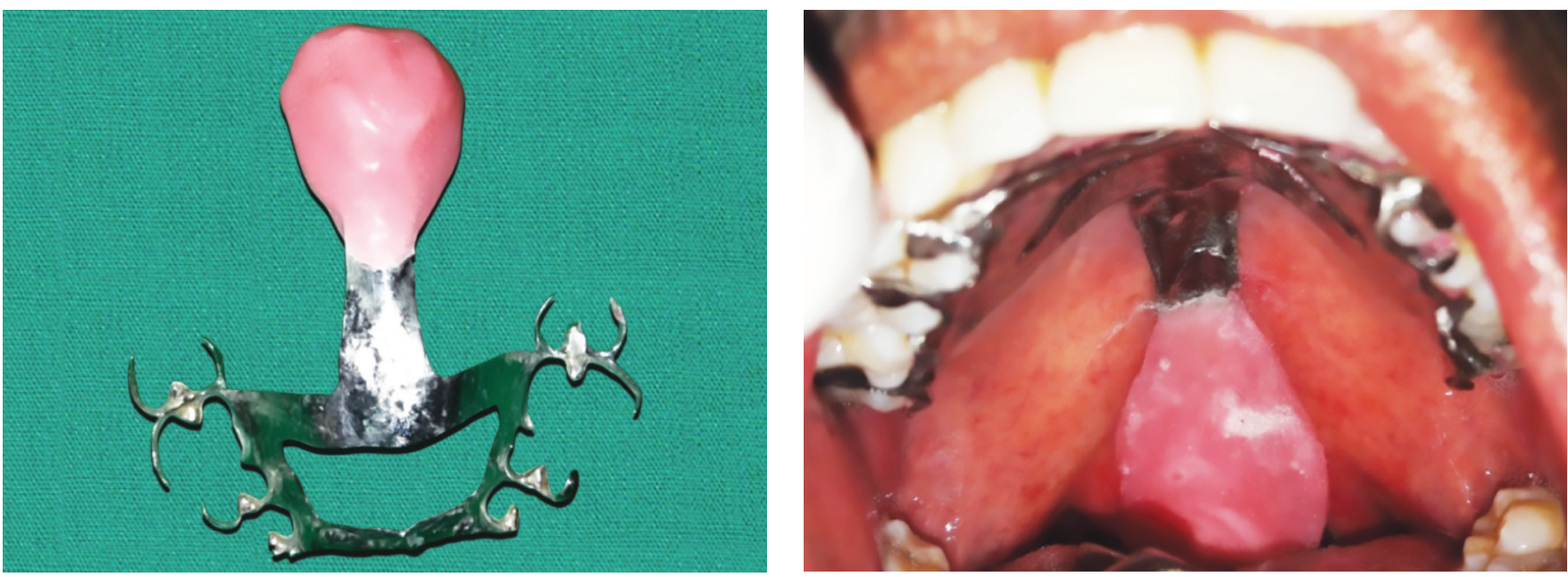

Figs 6A and B: (A) Final finished and polished speech aid prosthesis; (B) Speech aid prosthesis inserted

role in the improvement and teach them how to practice phonation with the presence of prosthesis.

\section{CONCLUSION}

The speech-aid prostheses is a viable treatment approach for patients suffering from velopharyngeal incompetency, which cannot be surgically treated, or when the subject in non-compliant with surgical intervention. Functional impression and molding around defect are essential for maximizing the effect of velopharyngeal closure.

For better results, maintenance of the prosthesis and oral hygiene is of great importance. The prosthesis being removable needs to be cleaned regularly and polished to prevent the accumulation of plaque. In the present case, the appreciable amount of speech improvement was found with a follow-up of 2 years. Consonants (e.gp,b,t,d) earlier had hypernasality became clear after adaptation period. Hence patient speech intelligibility increased.

\section{CLINICAL SIGNIFICANCE}

The enhanced role of CAD-CAM laser sintering technology in the proper management of VI by fabricating efficient SAP. With the use of CAD-CAM laser sintering technology, the fabricated SAP has a high degree of precision and predictability in comparison to the regular mode of casting procedures. It not only results in a better fit but also improved the quality of phonetics due to better sealing and muscle response.

\section{REFERENCES}

1. Mahanna GK, Beukelman DR, Marshall JA, Gaebler CA, Sullivan M. Obturator prostheses after cancer surgery: an approach to speech outcome assessment. J Prosthet Dent. 1998 Mar 31;79(3):310-316.

2. Turkaslan S, Baykul T, Aydin MA, Ozarslan MM. Articulation performance of patients wearing obturators with different buccal extension designs. Eur J Dent. 2009 Jul;3(3):185.

3. Knowles JC, Chalian VA, Shanks JC. A functional speech impression used to fabricate a maxillary speech prosthesis for a partial glossectomy patient. J Prosthet Dent 1984;51: 232-237.

4. Davis JW, Lazarus C, Logemann J, Hurst PS. Effect of a maxillary glossectomy prosthesis on articulation and swallowing. J Prosthet Dent. 1987;57(6):71

5. Skolnick L, McCall GN, Barnes M. The sphincteric mechanism of velopharyngeal closure. Cleft Palate J. 1973;10:286-305.

6. Shifman A, Finkelstein Y, Nachmani A, Ophir D. Speech-aid prostheses for neurogenic velopharyngeal incompetence. The Journal of prosthetic dentistry. 2000 Jan 1;83(1):99-106.

7. Abreu A, Levy D, Rodriguez E, Rivera I. Oral rehabilitation of a patient with complete unilateral cleft lip and palate using an implant-retained speech-aid prosthesis: Clinical report. Cleft Palate Craniofac J. 2007;44:673-677.

8. Kummer AW, Speech Evaluation for Patients with Cleft Palate. Clin Plastic Surg. 2014;41: 241-251

9. Tobey EA, Lincks J. Acoustic analyses of speech changes after maxillectomy and prosthodontic management. J Prosthet Dent. 1989 Oct 31;62(4):449-455.

10. Beumer J, Curtis A, Marunick MT. Maxillofacial rehabilitation. Prosthodontic and surgical considerations. St Louis: Ishiyaku Euro-America; 1996. p. 285-329.

11. Chambers MS, Lemon JC, Martin JW. Obturation of the partial soft palate defect. J Prosthet Dent. 2004;91(1):75-79.

12. Taylor TD, Desjardins RP. Construction of the meatus-type obturator: its advantages and disadvantages. J Prosthet Dent. 1983;49(1):80-84.

13. Alageel O, Abdallah MN, Alsheghri A, Song J, Caron E, Tamimi F. Removable partial denture alloys processed by laser-sintering technique. J Biomed Mater Res B Appl Biomater 2017;106:1174-1185. 